\title{
Predicción de la accidentalidad laboral en la industria de pulpa y papel usando algoritmos de clasificación
}

\author{
Rodolfo Mosquera ${ }^{\text {* }}$, Liliana Parra ${ }^{2}$, Ana J Ledesma ${ }^{3}$ y Héctor F Bonilla ${ }^{4}$ \\ (1) Universidad de San Buenaventura-Cali-Facultad de Ingeniería - Nuevas tecnologías trabajo y gestión-Carrera 122 \# \\ 6-65, Cali, 760031 - Colombia (correo-e: rmosqueran@usbcali.edu.co). \\ (2) Universidad Libre, Sede Bogotá, Facultad de Derecho, Centro de Investigaciones Socio jurídicas, Grupo de \\ investigación Estudios Interdisciplinarios DESC y el Mundo del Trabajo. Colombia \\ (correo-e: liliana.parrao@unilibre.edu.co). \\ (3) Universidad Icesi, Cali, Colombia (correo-e: ajledesma@icesi.edu.co). \\ (4) Pontificia Universidad Javeriana, Facultad de Ingeniería, Departamento de Ingeniería Civil e Industrial, Cali, \\ Colombia (correo-e: hector.bonilla@javerianacali.edu.co).
}

* Autor a quien debe ser dirigida la correspondencia

Recibido Jul. 24, 2020; Aceptado Sep. 24, 2020; Versión final Oct. 20, 2020, Publicado Feb. 2021

\section{Resumen}

En este artículo se propone un sistema de clasificación para la identificación y la prevención de accidentes laborales en las bodegas de almacenamiento de fibra en una empresa de pulpa de papel. Eso se hace con base en el análisis de variables que incluyen la circulación de peatones, bobcat, tractocamiones, acceso, zonas de circulación peatonal y barandas. La metodología propuesta define y entrena el sistema propuesto con datos recopilados en planta respecto a accidentalidad e incidentes laborales. Se compararon los resultados de tres algoritmos: redes bayesianas, naive bayes y árboles de decisión. Los resultados muestran que, en el $90 \%$ de los casos, el clasificador de arboles de decisión J48 identifica correctamente los casos posibles de accidentalidad laboral. Se concluye que la identificación de los atributos que generan la ocurrencia de incidentes y accidentes laborales, permite generar un árbol de decisión C4.5 (J48) que sirve de herramienta y soporte para labores de prevención de accidentes laborales.

Palabras clave: minería de datos; redes bayesianas; naive; árboles de decisión; pulpa; papel; accidentalidad laboral

\section{Applying data mining techniques to predict occupational accidents in the pulp and paper industry}

\begin{abstract}
This research study proposes a classification system to identify and prevent occupational accidents in fiber storage warehouses at a pulp and paper facility. The present analysis is based on variables including pedestrian circulation, bobcat, trailer trucks, access, pedestrian circulation zones, and handrails. The proposed methodology defines and trains the system by using occupational accident event data collected at the facility. Three different predicting algorithms are used: J48 decision-making trees, Naive Bayes, and Bayesian nets. The results show that the $\mathbf{J} 48$ decision-making tree algorithm accurately identifies possible occupational accidents $90 \%$ of the time. It is concluded that identifying variables involved in occupational accidents allows generating a C4.5 (J48) decision-making tree that can be used as a support tool to prevent occupational accidents.
\end{abstract}

Keywords: data mining; bayesian nets; naive; decision-making trees; pulp; paper; occupational accidents 


\section{INTRODUCCIÓN}

Los accidentes laborales en la industria son un factor de consideración, puesto que pueden llegar a cobrar muchas vidas por la falta de control y gestión de los peligros y riesgos. Los modelos teóricos sobre la gestión del riesgo desde el análisis estadístico de los accidentes de trabajo y el control del perdidas existe desde la década de los 60 (Bird y Germain, 1996). Algunos accidentes industriales han marcado el direccionamiento de la gestión del riesgo, por ejemplo, el caso de la industria química de Bhopal en la india (Broughton, 2005), con 3.800 muertes ha sido uno de los más trágicos accidentes industriales en la historia. En el mundo algunos casos de accidentalidad laboral en la industria de pulpa y papel se han reportado, por ejemplo, los países del sudeste asiático, Malasia ha sido clasificada con la tercera tasa más baja de accidentes $(14,000$ casos) y de mortalidad (18.3\%), después de Brunei (con 7,658 casos de accidentes, $10 \%$ de mortalidad) y Singapur (7,452 casos de accidentes, con una tasa de fatalidad de 9.8\%) (Ahmad, 2017).

Diferentes procesos industriales han utilizado técnicas de minería de datos para analizar las causas de accidentes ocupacionales (Fragiadakis et al., 2014; Sanmiquel et al., 2015; Sarkar et al., 2019; Tixier et al., 2016), como por ejemplo, la industria petroquímica (Cheng et al., 2013), la industria minera en España (Sanmiquel et al., 2015, 2018), en la industria de la construcción también se han hecho aplicaciones para predecir la accidentalidad ocupacional (Cho et al., 2017), investigadores utilizaron técnicas de minería de datos para analizar la accidentalidad ocupacional en la industria manufacturera Turca basados en la relación entre días de trabajo perdidos, estado del trabajador posterior al accidente y el tipo de accidente (Altunkaynak, 2018), en la industria de manufactura Turca, también, se han analizado entre los años 2013 a 2016, un total de 242.537 accidentes ocupacionales utilizando técnicas de minería de datos para identificar los 5 principales factores causantes de accidentalidad (Mutlu y Altuntas, 2019), los investigadores han utilizado las técnicas de minería de datos para actualizar los métodos de análisis de patrones existentes a través de tres nuevos tipos de análisis (i) generación de conjuntos de elementos frecuentes temporales (T-FIG) para estudiar el efecto del tiempo en los patrones, (ii) generación de conjuntos de elementos de gravedad elevada (ESIG) para examinar la reducción del riesgo debido a sistemas seguros de control de riesgos en el modelo de Cero accidentes (Singh y Maiti, 2020). El estudio sobre estimaciones globales de accidentes laborales y enfermedades relacionadas con el trabajo 2017, declara que a nivel global ocurren 2.78 millones de muertes anuales, de donde el $13.7 \%$ se deben a accidentes ocupacionales mortales; las lesiones ocupacionales a nivel mundial ocupan un 14\% (Hämäläinen et al., 2017).

En Finlandia se han llevado a cabo estudios donde tratan de explicar la relación que existe entre la frecuencia de la accidentalidad y el riesgo al cual puede estar expuesto el trabajador forestal; este sector es uno de los más importantes a nivel económico para los finlandeses al lado de la industria metalúrgica (Salminen et al., 1999). Algunos estudios en la industria papelera se han centrado en identificar problemas de accidentalidad en países como Canadá, donde los accidentes que se han presentado son debido a partes móviles de maquinaria, falta de protección, ausencia de procedimientos de bloqueo, inexperiencia de los trabajadores, falta de salvaguardas, falta de evaluación de riesgos, falta de supervisión, diseño de maquinaria deficiente, métodos de trabajo inseguro, pocas instrucciones claras para los trabajadores sobre cómo intervenir de manera segura en la maquinaria (Chinniah, 2015), lo que ha conllevado a formular estrategias para el control de la accidentalidad.

La industria de pulpa y papel en los países desarrollados ha prestado a través de los años especial interés a la gestión de los riesgos en los procesos (Costigane, 1920), en los países en desarrollo se hace necesario el análisis de los procesos y la generación de los controles para gestionar adecuadamente el riesgo. En la industria de pulpa y papel en Colombia los problemas de accidentalidad han sido poco estudiados, específicamente los que relacionan a trabajadores peatones y vehículos de transporte de materiales y de carga dentro de las instalaciones de las plantas de manufactura.

Al revisar la literatura disponible se encuentra que la mayoría de aplicaciones de minería de datos está referida al mejoramiento de las operaciones y de los procesos de transformación de la pulpa de madera en papel (por ejemplo: el blanqueo) (Pavan et al., 2006), algunos autores utilizan la minería de datos para revelar las intensidades de emisión de gases de efecto invernadero de diferentes productos de pulpa y papel (Nabinger et al., 2019) y muy pocas hacen referencia al tema en cuestión, la accidentalidad laboral. Otros autores han aplicado la minería de datos (Mosquera et al., 2016) y diferentes técnicas de inteligencia artificial (Mosquera et al., 2018a, 2018b) para clasificar riesgos de índole psicosocial, con buenos resultados en las aproximaciones. En atención a esta problemática, el presente estudio propone una metodología predictiva para identificar posibles accidentes laborales en los almacenes de fibra de pulpa y papel utilizando minería de datos. Las técnicas inteligentes permiten tomar variables que están presentes en los ambientes laborales de la industria, principalmente en almacenes y patios donde se almacena la fibra de pulpa de papel y donde por el tipo de actividad existen riesgos de accidentalidad a peatones por la interacción con vehículos. 


\section{MATERIALES Y MÉTODOS}

Esta sección presenta la información de la base de datos a intervenir y las descripciones cortas de tres modelos de clasificación representativos: Redes Bayesianas, Naïve Bayes y Árboles de Decisión (J48). En la figura 1 se presenta una visión general del modelo propuesto.

\section{Paso 1 y 2. Datos a Intervenir}

El conjunto de datos analizado proviene de datos recopilados en planta de situaciones donde se presentaron 72 incidentes y 28 accidentes laborales entre 2017 y 2018, para un total de 100 datos, como se muestra en la figura 2, se verifican los datos y se corrigen posibles errores. En la matriz general se presentan ocho interacciones posibles en las bodegas de almacenamiento de pulpa de papel. Los actores son: peatón, montacargas (bobcat), grúa hidráulica, tractocamión, bulldozer, tractomula, bus y vehículo particular. Para evaluar estas interacciones se define el grado de peligro, el cual está compuesto por: la frecuencia, probabilidad y severidad del grado de ocurrencia del evento. Se evalúan también las condiciones de seguridad presentes en la planta denominado controles existentes y los controles faltantes con respecto a elementos de protección personal, elementos de seguridad industrial y elementos de seguridad vial. Esta evaluación determina el grado de riesgo al cual está expuesto el trabajador en las bodegas de almacenamiento, el grado de riesgo se define como, grado de riesgo aceptable, riesgo menor y riesgo alto. Los controles existentes y faltantes se evalúan sobre las siguientes condiciones: Cebra-camino peatón, acceso peatón, señal "Zona Restringida", señal "Tránsito Peatón", sirena, "Baranda/cadena", Chaleco, Espejos en pasillos circulación y policía.

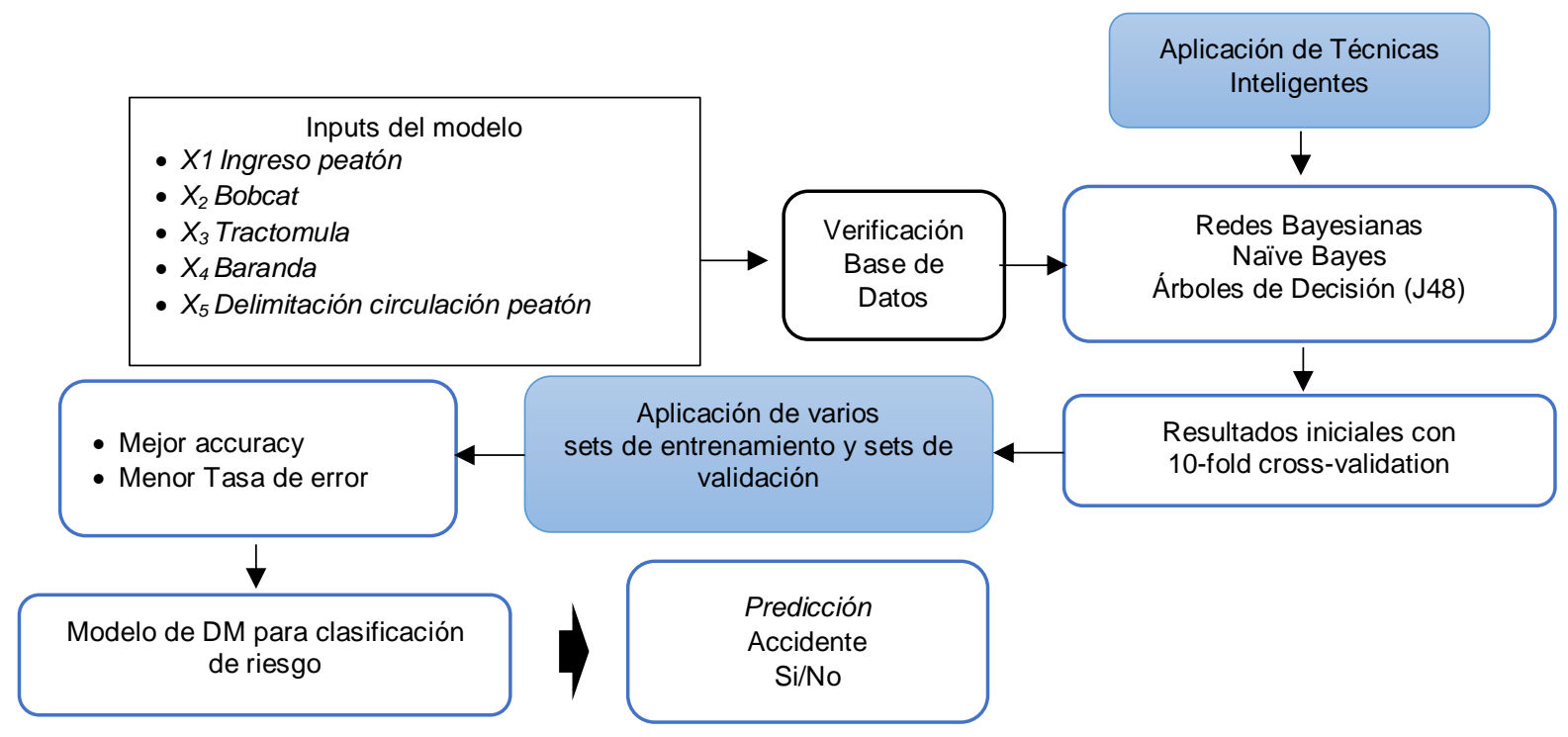

Fig. 1. Visión general del modelo propuesto.

\section{Paso 3. Variables de seguridad industrial}

De acuerdo con la información recolectada en el tiempo de observación de los eventos, teniendo en cuenta las variables contenidas en controles existentes y controles faltantes y los tipos de interacción, se decidió desarrollar un modelo que contara con las variables de control del proceso de accidentalidad en la zona de almacenamiento para aplicar modelos de minería de datos (se utilizó la herramienta de minería de datos WEKA V.3.8.4) (Hall et al., 2009) y apoyar la clasificación del riesgo. Para el computo de los modelos se utilizó un equipo con procesador Intel Core i7, 3.1 GHz, 8GB RAM.

Las variables con las cuales se desarrolla la metodología son: a) Peatón: El peatón realiza o no ingreso a la zona de almacenamiento y a las zonas de circulación de vehículos teniendo algún tipo de interacción con estos; b) Bobcat: Existe interacción con el peatón o circulación de bobcat en el área de almacenamiento; c) Tractomula: Existe interacción con el peatón en el área de almacenamiento; d) La baranda de seguridad que prohíbe el ingreso del bobcat a las áreas de circulación mientras existan peatones realizando interacción en el área de almacenamiento se encuentra activa; e) Existe delimitación de pasillos en las áreas de circulación de peatones; f) Accidente: La respuesta a la intervención de las variables en una probable situación de las condiciones antes expuestas es Si/No existe accidente. 


\section{Paso 4. Algoritmos}

Una red bayesiana o modelo probabilístico en un grafo acíclico es un modelo probabilístico de grafos que representa un conjunto de variables aleatorias y sus dependencias condicionales a través de un grafo acíclico dirigido (Niculescu-Mizil y Caruana, 2007). Dado un conjunto de datos (variables de riesgo) $=\left\{X_{1}, \ldots, X_{m}\right\}$ donde cada $x^{i}$ es una asignación completa de las variables de riesgo asignado $X_{1}, \ldots, X_{100}$, es posible construir tanto la estructura $G$ como los parámetros $\theta$. Siguiendo el paradigma bayesiano, la probabilidad posterior de la estructura dada la información se estima a través de las reglas de Bayes.

\begin{tabular}{|c|c|c|c|c|c|c|c|c|c|c|c|c|c|c|c|c|c|c|c|}
\hline \multirow[b]{2}{*}{ Ítem } & \multirow[b]{2}{*}{ Área } & \multirow[b]{2}{*}{ Descripción } & \multicolumn{3}{|c|}{ Tipo de Interacción } & \multicolumn{4}{|c|}{ GP (Grado de Peligro) } & \multicolumn{5}{|c|}{$\begin{array}{l}\text { Controles } \\
\text { Existentes }\end{array}$} & \multicolumn{4}{|c|}{$\begin{array}{l}\text { Controles } \\
\text { Faltantes }\end{array}$} & \multirow[b]{2}{*}{ Estatus } \\
\hline & & & 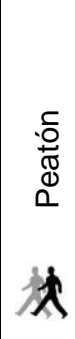 & $\begin{array}{l}\overrightarrow{1} \\
0 \\
0 \\
0 \\
0 \\
\infty \\
\\
\end{array}$ & 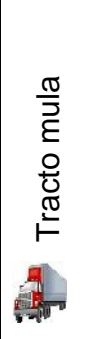 & 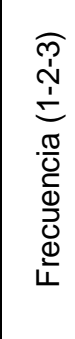 & 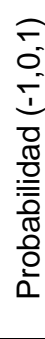 & 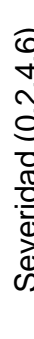 & 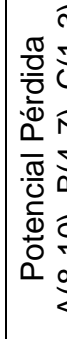 & 产 & 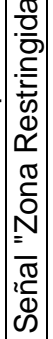 & $\stackrel{\substack{c \\
\Phi}}{\bar{\omega}}$ & 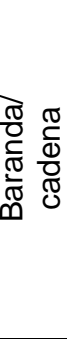 & $\begin{array}{l}\circ \frac{0}{D} \\
\dot{0} \\
w \\
w\end{array}$ & 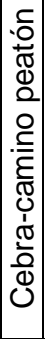 & 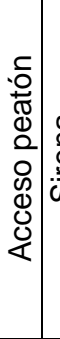 & & 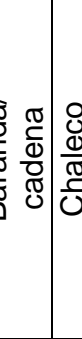 & \\
\hline 13 & $\begin{array}{l}\mathcal{C} \\
\stackrel{\mathcal{D}}{\mathscr{E}} \\
\stackrel{\mathscr{E}}{\tau} \\
\end{array}$ & $\begin{array}{c}\text { MP - pasillo de } \\
\text { muelles de cargue / } \\
\text { descargue }\end{array}$ & $X$ & X & & 2 & 1 & 6 & 9 & & 5 & & & 3 & 4 & & 4 & 4 & $\begin{array}{l}\text { Riesgo } \\
\text { Alto }\end{array}$ \\
\hline 14 & 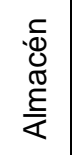 & $\begin{array}{c}\text { MP - zona entre } \\
\text { patio de maniobras } \\
\text { y taller de } \\
\text { montacargas }\end{array}$ & $\mathrm{X}$ & X & 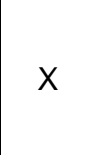 & 1 & 1 & 6 & 8 & & & & & & & 4 & & & $\begin{array}{l}\text { Riesgo } \\
\text { Menor }\end{array}$ \\
\hline
\end{tabular}

Fig. 2. Ejemplo de análisis de interacción peatones vehículos y riesgo de accidentalidad

$$
\text { "P(G|D)œP(G)P(D|G)" }
$$

Donde, $P(G)$ indica los valores iniciales de las variables de riesgo donde se cree que la estructura $G$ es correcta, si no hay razón para preferir una estructura sobre otra, se debe asignar la misma probabilidad a todas las estructuras. Si existe un orden conocido en los nodos en todos los padres de un nodo lo preceden en el orden, un primer valor puede evaluarse especificando la probabilidad de que cada uno de los arcos posibles $(n-1) / 2$ esté presente en la estructura correcta y logre clasificar la mejor probabilidad.

\section{Naïve Bayes}

Naïve Bayes es un clasificador probabilístico fundamentado en el teorema de Bayes. En el clasificador Bayer (la función Classify) (Perikos y Hatzilygeroudis, 2016) se define como:

$$
" p\left(X_{1}=x_{1}, \ldots, X_{n}=x_{n} \mid C=c\right)=\prod_{i=1}^{n} \rho\left(X_{i}=x_{i} / C=c\right) "
$$

Dónde, las $n$ variables predictoras de riesgo $X_{1}, \ldots, X_{100}$ son continuas, se tiene que para hallar el valor de la variable $C$, que denotamos por $C$, que maximiza la probabilidad a posteriori de la variable $C$, dada la evidencia expresada como las variables de riesgo $X_{1}, \ldots, X_{100}$, esto es, " $X=\left(X_{1}, \ldots, X_{100}\right)$." Por tanto, en el paradigma Naïve Bayes, la búsqueda de la predicción más probable, $C^{*}$, conocidos los valores de las variables de riesgo $\left(X_{1}, \ldots, X_{113}\right)$ de un determinado individuo en riesgo, (Perikos y Hatzilygeroudis, 2016) se reduce a:

$$
\begin{aligned}
& \text { "C* } \arg _{C} \max \rho\left(C=c \mid X_{1}=x_{1}, \ldots, X_{n}=x_{n}\right) " \\
& "=\arg _{c} \operatorname{máx} p(C=c) \prod_{i=1}^{n} \rho\left(X_{i}=x_{i} / C=c\right) "
\end{aligned}
$$




\section{Árboles de Decisión}

Los datos de entrenamiento de riesgos ocupacionales $S=s_{1}, s_{2}, \ldots s_{100}$ de datos ya clasificados. Cada valor $s_{i}=\left\{X_{1}, X_{2}, \ldots, X_{100}\right\}$ es un vector donde $x_{1}, X_{2}, \ldots X_{100}$ representan las características de los riesgos presentes en la bodega de almacenamiento de la planta que son factores que pueden producir un accidente. Los datos de entrenamiento son incrementados con un vector $Z=\left\{z_{1}, z_{2}, \ldots z_{100}\right\}$ donde $z_{1}, z_{2} \ldots, z_{100}$ representan la clase a la que pertenece cada dato (Quinlan y Salzberg, 1994). Para el modelo se tomaron 100 variables como predictores y una variable de clase como predictora de la ocurrencia del accidente.

\section{Paso 5. Datos de validación, entrenamiento y prueba}

Se ejecutaron los modelos con 10-fold cross-validation en primera instancia y se obtienen los resultados, luego el conjunto de datos se divide en varios trainings set y test set, para observar con que parámetros clasifica mejor cada uno de los modelos y plantear este modelo como la metodología para predecir la ocurrencia de un accidente laboral. La métrica usada para la clasificación es el accuracy.

\section{Paso 6. Análisis de estabilidad}

Para garantizar la estabilidad del sistema en los resultados, se realizó un análisis de varianza usando la ecuación (14). En este análisis, $Y_{i}$ representa la respuesta a las variables; $T_{i}$, el efecto causado por el tratamiento $i$-esimo y $\varepsilon_{i}$, el $i$-esimo error experimental. La información debe cumplir los requerimientos de independencia y normalidad requeridos. El análisis se realizó con un intervalo de confianza del $99.5 \%$.

$$
" Y_{i}=\mu+T_{i}+\varepsilon_{i} "
$$

\section{RESULTADOS}

Se organizó la información acorde a las respuestas de cada atributo en el dataset, se realizó la aplicación de técnicas de Aprendizaje automático, en primera instancia con 10-fold cross-validation, luego se particionó el conjunto de datos y se obtuvieron los resultados, se seleccionó el mejor modelo para la predicción y por último el análisis de estabilidad de los resultados.

\section{Paso 1 y 2. Datasets y comprobación de la información}

Se definieron las variables a manejar para la predicción del riesgo asociado (Tabla 1), en total 5 variables, con valores cualitativos y 100 registros, de los cuales 100 son variables predictoras y 1 variable de clase.

Tabla 1. Definición de variables

\begin{tabular}{|l|l|}
\hline Variables & Definición \\
\hline Peatón & Ingresó/No ingresó \\
\hline Bobcat & Interacción/No interacción \\
\hline Tractomula & Interacción/No interacción \\
\hline Baranda & True/False \\
\hline Delimitación Circulación Peatón & Existe/No existe \\
\hline Accidente & Sí/No \\
\hline
\end{tabular}

\section{Paso 3. Variables de Seguridad Industrial}

La definición ingreso o no ingreso de la variable peatón, se produce si el peatón tiene acceso al patio de almacenamiento. Las variables bobcat y tractomula hacen referencia a que, si se encuentra el peatón en el área de almacenamiento, se encuentre algún tipo de vehículo bobcat o tractomula circulando en el patio. La definición true o false de la variable baranda hace referencia a que si la medida de seguridad que controla el ingreso de un vehículo al patio (bobcat o tractomula) fue activada o no, teniendo en cuenta si hay circulación de peatones en el patio. La definición de la variable delimitación de circulación de peatones hace referencia a si se encuentran señalizadas las zonas por donde deben y no deben circular peatones y se cumple con esta regla. La información de base de datos y archivos para el desarrollo de este artículo puede ser consultado aquí (Mosquera, 2020): https://zenodo.org/record/3697323\#.XmJnp0q23IX 


\section{Pasos 4 y 5. Aplicación de técnicas de Aprendizaje automático}

Se aplicó en primera instancia los algoritmos Redes Bayesianas, Naïve Bayes y Árboles de Decisión al conjunto de datos aplicando 10-fold cross-validation, se obtuvieron los resultados que se muestran en la tabla 2, la cual presenta los resultados generados por cada algoritmo.

Tabla 2. Comparación entre algoritmos de clasificación aplicando 10-fold cross-validation. ( ${ }^{*}$ accuracy)

\begin{tabular}{|c|c|c|c|c|c|c|}
\hline \multirow{2}{*}{ Test } & \multicolumn{2}{|c|}{ Redes Bayesianas } & \multicolumn{2}{c|}{ Naïve Bayes } & \multicolumn{2}{c|}{ Árboles de Decisión } \\
\cline { 2 - 7 } & $\begin{array}{c}\% \\
\text { Clasificación }\end{array}$ & $\begin{array}{c}\% \text { Tasa } \\
\text { Error }\end{array}$ & $\begin{array}{c}\% \\
\text { Clasificación* }\end{array}$ & $\begin{array}{c}\% \text { Tasa } \\
\text { Error }\end{array}$ & $\begin{array}{c}\% \\
\text { Clasificación* }\end{array}$ & $\begin{array}{c}\% \text { Tasa } \\
\text { Error }\end{array}$ \\
\hline $\begin{array}{c}10-\text { fold cross- } \\
\text { validation }\end{array}$ & 83 & 17 & 83 & 17 & 87 & 13 \\
\hline
\end{tabular}

Los resultados muestran en este caso que el porcentaje de clasificación y la tasa de error de los árboles de decisión (J48) sembrando 1 semilla para las simulaciones (random seed for XVAL/\% split) mayor y menor respectivamente, en comparación con los algoritmos de Redes Bayesianas y Naïve Bayes, con un $87 \%$ de correcta clasificación y una tasa de error del 13\% frente al $83 \%$ y $17 \%$ respectivamente de cada uno. En segunda instancia se realizaron pruebas al conjunto de datos con sets de entrenamiento y sets de validación para observar la mejor clasificación con cada algoritmo como se muestra en la tabla 3.

Tabla 3. Comparación entre algoritmos de clasificación (*Accuracy)

\begin{tabular}{|c|c|c|c|c|}
\hline $\begin{array}{c}\% \\
\text { Entrenamiento }\end{array}$ & \% Validación & $\begin{array}{c}\text { \% Clasificación }{ }^{*} \text { Tasa } \\
\text { Error Redes Bayesianas }\end{array}$ & $\begin{array}{c}\text { \% Clasificación } \\
\text { Error Naïve Bayes }\end{array}$ & $\begin{array}{c}\text { \% Clasificación }{ }^{*} \text { Tasa } \\
\text { Error Árboles de Decisión }\end{array}$ \\
\hline 20 & 80 & $76,25 / 23,75$ & $76,25 / 23,75$ & $71,25 / 28,75$ \\
\hline 40 & 60 & $76,66 / 23,33$ & $83,33 / 16,66$ & $83,33 / 16,66$ \\
\hline 50 & 50 & $90 / 10$ & $90 / 10$ & $90 / 10$ \\
\hline 60 & 50 & $87,5 / 12,5$ & $87,5 / 12,5$ & $90 / 10$ \\
\hline 70 & 30 & $86,66 / 13,33$ & $86,66 / 13,33$ & $90 / 10$ \\
\hline 80 & 20 & $80 / 20$ & $80 / 20$ & $85 / 15$ \\
\hline & Promedio Simple & $82,84 / 17,15$ & $82,84 / 17,15$ & $84,93 / 15,07$ \\
\hline
\end{tabular}

Los resultados muestran que los árboles de decisión con un 84,93\% en promedio de correcta clasificación y una tasa de error del $15,07 \%$, son el algoritmo que tiene mejor aproximación a la predicción de los resultados de accidentalidad laboral. Desde esta perspectiva, se decidió abordar y diseñar el modelo junto a la metodología propuesta. Teniendo en cuenta que los árboles de decisión dan excelentes resultados de clasificación se decidió graficar el respectivo modelo (Figura 3) acorde a los resultados hallados en la tabla 2 para que sirva de soporte a las personas encargadas de realizar inspecciones en materia de Seguridad y Salud en el Trabajo en las áreas de almacenamiento de fibra de papel en las plantas de producción de pulpa y papel.

Con los mejores resultados obtenidos del test de entrenamiento y del test de validación del algoritmo árboles de decisión, se realizaron 5 réplicas de cada uno para observar la estabilidad de los resultados de cada modelo. A estas diferentes pruebas con el algoritmo se le consideran tratamientos, lo que a posterior nos permitirá probar si el modelo es significativo o no. Se realizaron 5 tratamientos, debido que a medida que se realizaba cada una de las corridas los resultados fueron iguales (Tabla 4). Los valores aquí obtenidos se utilizaron para comprobar la estabilidad del sistema.

Tabla 4. Replicas con cada algoritmo y su promedio general.

\begin{tabular}{|c|c|c|c|c|c|}
\hline Técnica & \multicolumn{5}{c|}{ Réplicas } \\
\hline $\begin{array}{c}1 \text { semilla. Partición de la base de datos. } \\
\text { Entrenamiento (50\%) y Validación (50\%) }\end{array}$ & 89,9 & 90 & 90 & 90 & 90 \\
\hline $\begin{array}{c}1 \text { semilla. Partición de la base de datos. } \\
\text { Entrenamiento (60\%) y Validación (40\%) }\end{array}$ & 90 & 89,9 & 90 & 90 & 89,98 \\
\hline $\begin{array}{c}1 \text { semilla. Partición de la base de datos. } \\
\text { Entrenamiento (70\%) y Validación (30\%) }\end{array}$ & 89,8 & 90 & 90 & 89,9 & 90 \\
\hline
\end{tabular}


El árbol de decisión permite observar que la principal variable que incide directamente sobre la accidentalidad de los peatones en los patios de almacenamiento es el bobcat, seguido por el accionar de la baranda y la presencia de tractomulas, la delimitación de los pasillos por donde pueden circular los peatones también son un factor importante al momento de analizar incidentes y accidentes.

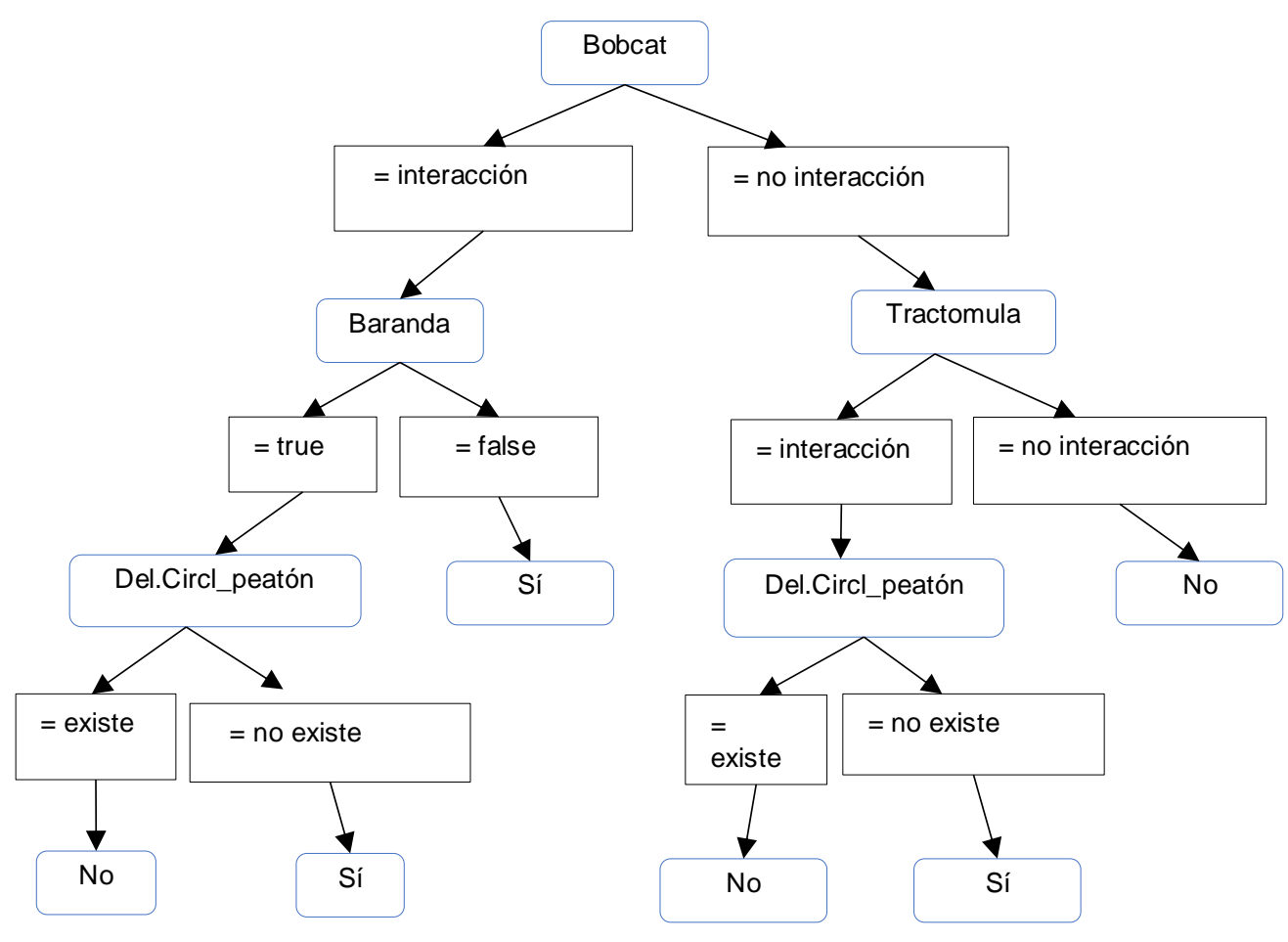

Fig. 3. Árbol de decisión para la predicción de accidentes laborales en el área de almacenamiento de fibra de papel

\section{Paso 6. Análisis de estabilidad}

Tomando como referencia las soluciones encontradas en el paso 5 (Tabla 4), se realizó el análisis de varianza, bajo un intervalo de confianza del 99.5\%. Dado que en la Tabla 6 , el $\mathrm{F}$ tabulado es mayor que el $\mathrm{F}$ calculado, se puede concluir que el modelo no es significativo; es decir, bajo un intervalo de confianza del $99.5 \%$ los datos son estadísticamente iguales.

Tabla 6. Análisis de varianza (Anova).

\begin{tabular}{|l|l|l|l|l|l|}
\hline \multicolumn{1}{|c|}{ Fuente Variación } & \multicolumn{1}{|c|}{ SS } & \multicolumn{1}{c|}{ df } & \multicolumn{1}{c|}{ MS } & F & Prob>F \\
\hline Columnas & 0,278 & 4 & 0,069 & 0,27 & 0,8921 \\
\hline Error & 2,601 & 10 & 0,26 & & \\
\hline Total & 2,88 & 14 & & & \\
\hline
\end{tabular}

\section{DISCUSIÓN}

A través del uso de técnicas de inteligencia artificial para la clasificación, se pudo identificar atributos que influyen en la probabilidad de ocurrencia de un accidente laboral en bodegas de almacenamiento de fibra en la industria de pulpa y papel. En este artículo se muestra que, la influencia de los vehículos en los patios de almacenamiento son un factor predominante para la ocurrencia de accidentes a peatones, también, que el no seguir las normas y procedimientos en materia de seguridad vial por parte del peatón ocasiona accidentes. Se puede mostrar a través de la revisión de la literatura que investigadores están usando redes bayesianas para predecir las lesiones y posibles traumatismos que ocurren en los accidentes de tránsito utilizando las características de los conductores, las características de las vías y las características de los accidentes (de Oña et al., 2011) y otros investigadores han realizado aproximaciones a través del modelado de accidentes ocupacionales con redes bayesianas (García-Herrero et al., 2012), lo que permite inferir que el uso de modelos bayesianos actualmente son importantes tal como se evidenció en este artículo y que la aproximación a la identificación y clasificación de accidentalidad laboral a través de modelos inteligentes es viable. 
Sarkar et al. (2019) utilizaron algoritmos de Máquinas de Soporte Vectorial (SVM) y Redes Neuronales Artificiales (ANN) optimizando los datos de entrada con Algoritmos Genéticos (GA) y con Optimización por enjambre de partículas (PSO) logrando resultados de clasificación de $90.53 \%$ y $90.67 \%$ respectivamente, en éste sentido, los resultados en éste estudio, muestran resultados comparables a los obtenidos por ellos. Los resultados obtenidos por (Kang y Ryu, 2019) utilizando el algoritmo de Random Forest (RF) del $71.3 \%$ de precisión para la predicción de la accidentalidad en la industria de la construcción, muestra que la utilización de algoritmos a nivel de clasificación de accidentalidad laboral en diferentes industrias es de suma importancia, como se infiere también para la industria de pulpa y papel.

Los modelos de árboles de decisión en este tipo de variables son bastante efectivos, logrando clasificaciones bastante altas y que sirven de soporte para los encargados de los sistemas de gestión de la Seguridad y la Salud en el Trabajo en las empresas para la prevención de accidentes laborales. Cabe resaltar que el número de muestras obtenidas en el período de dos años se considera acorde a lo que se esperaría de una empresa de carácter multinacional, donde se encuentran implementados sistemas integrados de gestión en toda su estructura organizacional. Sin embargo, se puede observar que la muestra obtenida es estadísticamente buena para poder realizar la modelización de los atributos identificados que intervienen en la accidentalidad laboral de este tipo de industria.

Al realizar la comparación de los modelos de clasificación utilizados en este estudio (Tabla 2 y tabla3), se puede inferir que el uso de las técnicas de minería de datos para la identificación de accidentalidad laboral en este tipo de procesos es posible y el futuro desarrollo con nuevos algoritmos o la implementación con nuevas variables podría mejora la calidad de la predicción, también, se podría trabajar con técnicas que utilicen el procesamiento digital de imágenes en tiempo real para identificar posibles falencias en el proceso y que de manera automática se reconozcan las variables que pueden ocasionar un accidente laboral.

En este artículo, se identifica a través del uso de varias técnicas inteligentes, los principales patrones que inciden en la ocurrencia de incidentes y accidentes laborales en la bodega de almacenamiento de fibra de una empresa de pulpa y papel. Los resultados observados muestran que los factores más influyentes para que ocurra un incidente o un accidente son el tránsito de montacargas, la circulación de tractomulas, el no seguir las indicaciones de seguridad industrial, la falta de demarcación para que los peatones pueden circular en áreas de almacenamiento de fibra de pulpa de papel.

\section{CONCLUSIONES}

La identificación de los atributos que generan la ocurrencia de incidentes y accidentes laborales, permite generar un árbol de decisión C4.5 (J48) que sirve de herramienta y soporta las labores de prevención de accidentes laborales. También, se puede concluir que el modelo con mejor aproximación logró obtener una efectividad del $90 \%$. Se hace necesario el obtener más datos de otras empresas dedicadas a la transformación de pulpa para desarrollar papel que permita tener un dataset mucho más robusto. La obtención de modelos de minería de datos o de aprendizaje automático, que logren identificar y clasificar posibles incidentes y accidentes en la industria de pulpa y papel se hace necesaria para la prevención de estos fenómenos. Desde la Seguridad y la Salud en el Trabajo se concluye que se observan causas inmediatas como actos subestándares (falta de comunicación entre los Peatones), condiciones subestándares (como, no señalar, no advertencias de peligro). Causas básicas como, factores de trabajo. Como estándares deficientes de trabajo (manutención inadecuada de las normas) y falta de control (Falta de controles de ingeniería y administración del cambio) y falta de comunicaciones personales (no se comunican los peatones).

\section{AGRADECIMIENTOS}

Se expresa el agradecimiento a la Dirección de Investigaciones de la Universidad de San Buenaventura, Cali, por el apoyo prestado a este proyecto como resultado del proyecto de investigación: "Aplicaciones de la Inteligencia Artificial en la Seguridad y Salud en el Trabajo en el reconocimiento de Riesgos Laborales con fecha de inicio Septiembre 02 de 2019, el cual se desarrolló como parte del trabajo colaborativo entre el grupo de investigación Nuevas Tecnologías Trabajo y Gestión y el grupo Estudios Interdisciplinarios DESC y el Mundo del Trabajo de la Universidad Libre, Bogotá. Se expresa también agradecimientos a Colciencias por el apoyo a éste proyecto a través de la formación de alto nivel, gracias a la convocatoria de Doctorado Nacional -647 .

\section{REFERENCIAS}

Ahmad, S. Accident rate manufacturing plant: A case study at the pulp and paper industry in east coast Malaysia. Journal of Advanced Research in Social and Behavioural Sciences, ISSN: 2462-1951, 7(1), 10-19 (2017) 
Altunkaynak, B. A statistical study of occupational accidents in the manufacturing industry in Turkey. https://doi.org/10.1016/j.ergon.2018.02.012, International Journal of Industrial Ergonomics, 66, 101-109 (2018)

Bird, F.E., y Germain, G.L. Practical loss control leadership, 3rd ed, Det Norske Veritas, Loganville, GA, USA (1996)

Broughton, E. (2005). The Bhopal disaster and its aftermath: A review. https://doi.org/10.1186/1476-069X-4-6, Environmental Health, 4(1) (2005)

Cheng, C.W., Yao, H.Q., y Wu, T.C. Applying data mining techniques to analyze the causes of major occupational accidents in the petrochemical industry. https://doi.org/10.1016/j.jp.2013.07.002, Journal of Loss Prevention in the Process Industries, 26(6), 1269-1278 (2013)

Chinniah, Y. Analysis and prevention of serious and fatal accidents related to moving parts of machinery. https://doi.org/10.1016/j.ssci.2015.02.004, Safety Science, 75, 163-173 (2015)

Cho, Y., Kim, Y.C., y Shin, Y. Prediction Model of Construction Safety Accidents using Decision Tree Technique. https://doi.org/10.5345/JKIBC.2017.17.3.295, Journal of the Korea Institute of Building Construction, 17(3), 295-303 (2017)

Costigane, A.P. Accident Prevention in the Pulp and Paper Industry. https://www.jstor.org/stable/41972578, The Public Health Journal, 11(7), 307-315 (1920)

Fragiadakis, N.G., Tsoukalas, V.D., y Papazoglou, V.J. An adaptive neuro-fuzzy inference system (anfis) model for assessing occupational risk in the shipbuilding industry. https://doi.org/10.1016/j.ssci.2013.11.013, Safety Science, 63, 226-235 (2014)

Hall, M., Frank, E., y otros cuatro autores. The WEKA data mining software: An update. https://doi.org/10.1145/1656274.1656278, ACM SIGKDD Explorations Newsletter, 11(1), 10 (2009)

Hämäläinen, P., Takala, J., y Kiat, T.B. Global Estimates of Occupational Accidents and Work-related IIInesses $2017.1^{\mathrm{a}}$ Ed, 3-4. Published by Workplace Safety and Health Institute, Singapore, Singapore (2017)

Kang, K., y Ryu, H. Predicting types of occupational accidents at construction sites in Korea using random forest model. https://doi.org/10.1016/j.ssci.2019.06.034, Safety Science, 120, 226-236 (2019)

Mosquera, R. Pulp and paper risk accident classification in Colombia [Data set]. http://doi.org/10.5281/zenodo.3697323. Zenodo, (2020).

Mosquera, R., Castrillón, O.D., y Parra, L. Predicción de Riesgos Psicosociales en Docentes de Colegios Públicos Colombianos utilizando Técnicas de Inteligencia Artificial. https://doi.org/10.4067/S0718-07642018000400267, Información tecnológica, 29(4), 267-280 (2018a).

Mosquera, R., Castrillón, O.D., y Parra, L. Máquinas de Soporte Vectorial, Clasificador Naïve Bayes y Algoritmos Genéticos para la Predicción de Riesgos Psicosociales en Docentes de Colegios Públicos Colombianos. https://doi.org/10.4067/S0718-07642018000600153, Información tecnológica, 29(6), 153-162 (2018b)

Mosquera, R., Parra-Osorio, L., y Castrillón, O.D. Metodología para la Predicción del Grado de Riesgo Psicosocial en Docentes de Colegios Colombianos utilizando Técnicas de Minería de Datos. https://dx.doi.org/10.4067/S071807642016000600026, Información tecnológica, 27(6), 259-272 (2016)

Mutlu, N.G., y Altuntas, S. Assessment of occupational risks In Turkish manufacturing systems with data-driven models. https://doi.org/10.1016/j.jmsy.2019.09.008, Journal of Manufacturing Systems, 53, 169-182 (2019)

Nabinger, A., Tomberlin, K., Venditti, R., y Yao, Y. Using a Data-Driven Approach to Unveil Greenhouse Gas Emission Intensities of Different Pulp and Paper Products. https://doi.org/10.1016/j.procir.2018.12.001, Procedia CIRP, 80,689692(2019).

Niculescu-Mizil, A., y Caruana, R. Inductive Transfer for Bayesian Network Structure Learning. In: Proceedings of the Eleventh International Conference on Artificial Intelligence and Statistics, ISSN 1532-4435, 339-346 (2007)

Pavan, M., Todeschini, R., y Orlandi, M. Data Mining by Total Ranking Methods: A Case Study on Optimisation of the "Pulp and Bleaching" Process in the Paper Industry. https://doi.org/10.1002/adic.200690004, Annali Di Chimica, 96(1-2), 13-27 (2006)

Perikos, I., y Hatzilygeroudis, I. Recognizing emotions in text using ensemble of classifiers. https://doi.org/10.1016/j.engappai.2016.01.012, Engineering Applications of Artificial Intelligence, 51, 191-201 (2016)

Quinlan, J.R., y Salzberg, S.L. C4.5: Programs for Machine Learning by J. Ross Quinlan. Morgan Kaufmann Publishers, Inc., 1993. https://doi.org/10.1007/BF00993309, Machine Learning, 16(3), 235-240 (1994)

Salminen, S., Klen, T., y Ojanen, K. Risk taking and accident frequency among Finnish forestry workers. https://doi.org/10.1016/S0925-7535(99)00029-6, Safety Science, 33(3), 143-153 (1999)

Sanmiquel, L., Bascompta, y otros tres autores. Analysis of Occupational Accidents in Underground and Surface Mining in Spain Using Data-Mining Techniques. https://doi.org/10.3390/ijerph15030462, International Journal of Environmental Research and Public Health, 15(3),462(2018)

Sanmiquel, L., Rossell, J.M., y Vintró, C. Study of Spanish mining accidents using data mining techniques. https://doi.org/10.1016/j.ssci.2015.01.016, Safety Science, 75, 49-55 (2015) 
Sarkar, S., Vinay, S., y otros tres autores. Application of optimized machine learning techniques for prediction of occupational accidents. https://doi.org/10.1016/j.cor.2018.02.021, Computers \& Operations Research, 106,210-224 (2019)

Singh, K., y Maiti, J. A novel data mining approach for analysis of accident paths and performance assessment of risk control systems. doi:10.1016/jress.2020.10704. Reliability Engineering \& System Safety, 107041 (2020)

Tixier, A.J.P., Hallowell, M.R., Rajagopalan, B., y Bowman, D. Application of machine learning to construction injury prediction. https://doi.org/10.1016/j.autcon.2016.05.016, Automation in Construction, 69,102-114(2016) 